\title{
CHAMP Orbit Determination with GPS Phase- Connected, Precise Point Positioning
}

\author{
Sunil B. Bisnath and Richard B. Langley
}

Geodetic Research Laboratory, Department of Geodesy and Geomatics Engineering, University of New Brunswick, Fredericton, N.B., Canada

\section{Summary}

A completely geometric approach for precise orbit determination (POD) of low earth orbiters (LEOs) is presented, which does not rely on dynamic models, but only data from a GPS receiver onboard a LEO and the International GPS Service (IGS) GPS orbit and clock products. Initial processing of CHAMP receiver data indicated measurement anomalies requiring additional pre-processing. The excising of outliers becomes a significant concern in the processing, given that this results in significant gaps in datasets. Intermediate processing results indicate that orbit comparison precision approaching $30 \mathrm{~cm}$ r.m.s. in each position component is attainable. However, these results are severely impacted by the near ubiquitous data gaps in the preprocessed measurements. However, initial analysis of recent data indicates quality datasets capable of providing near-decimetre-level precision.

Keywords: orbit determination - GPS - point positioning

\section{Introduction}

The University of New Brunswick (UNB) has been performing GPS-based LEO orbit determination research since 1998. The investigations have centred on GPSonly solution strategies - referred to as geometric orbit determination (Bisnath and Langley 1999). The goals of the research were to answer the question: Can GPS alone be used to determine the precise orbit of a LEO, and if so, how well? With the removal of Selective Availability (SA), the objective evolved into POD with a single spaceborne GPS receiver. The CHAMP science satellite (GFZ 2001) and the geodetic-grade BlackJack receiver onboard represent an excellent opportunity to test these algorithms. 


\section{Methodology}

To make complete, efficient use of the available GPS data, the UNB processing strategy simultaneously utilizes the pseudorange measurements to compute LEO position and the carrier-phase measurements to compute LEO position change in a kinematic, sequential, least-squares filter/smoother - a state space approach. The primary inputs are: dual-frequency pseudorange and carrier-phase measurements from the LEO receiver; precise GPS constellation ephemerides; and precise constellation satellite clock offsets from GPS Time. The ephemerides and clock data are readily available from the IGS. The GPS-determined positions can be interpolated to produce a LEO state throughout the orbit. This processing flow is illustrated in Figure 1.

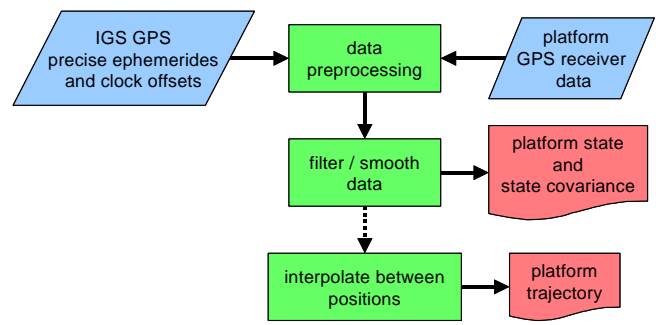

Fig. 1. Processing flow of the geometric orbit determination strategy

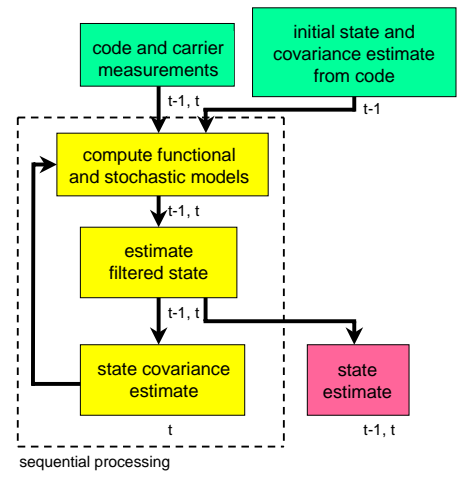

Fig. 2. Combination of pseudorange and carrier-phase observations in the kinematic, sequential least-squares filter

The parameterisation of the filter models is given in Bisnath and Langley (2001). A flowchart of the least-squares filter is shown in Figure 2. As can be seen, this filter is a variant of the code-smoothed-phase filter. Therefore a minimum number of continuous phase measurements are required for filter operation without re-initialisation. 
The technique then encompasses two philosophies: neither a reference receiver nor a reference receiver network is explicitly necessary, since that information is indirectly provided by the IGS data products - hence single receiver positioning; and no assumed dynamic models are required, since the time-differenced, carrierphase observations precisely measure motion - hence platform independence.

\section{Preprocessing}

It was found that the geometric CHAMP orbit solutions are very sensitive to data editing performed in the preprocessor. Data editing consists of applying a signalto-noise filter and a rate-of-change of widelane-phase minus narrowlanepseudorange linear combination filter. The former removes low strength signals at the measurement input stage, while the latter eliminates measurements that deviate from the norm before the initial estimation process. This preprocessing strategy therefore cleans the data in the measurement domain, without need for postestimation residual analysis or need for a reference CHAMP orbit to constrain the definition of typical measurement behaviour.

Figure 3 illustrates an example of data editing. The GPS satellite is tracked to almost $-15^{\circ}$, causing the signal-to-noise values to approach zero BlackJack units, and the rates-of-change of the linear combinations to deviate significantly from zero. The receiver satellite-tracking algorithm is responsible for this situation, producing large numbers of low elevation angle satellites tracked in the CHAMP anti-velocity direction. Measurements accumulated from these weak signals are deleted, resulting in intermittent poor data availability as can be seen in Figure 4.

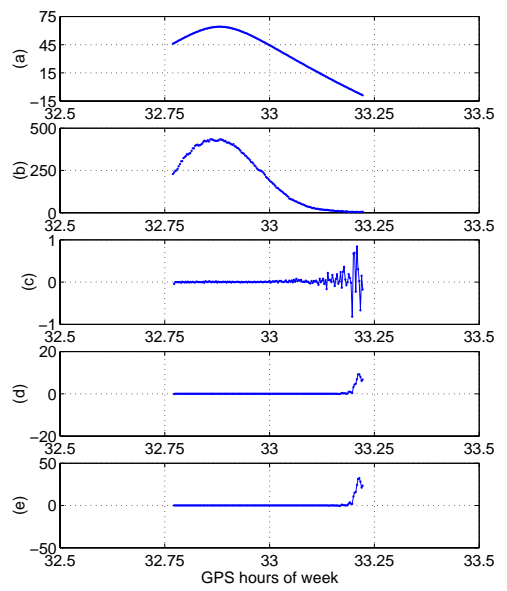

Fig. 3. Poor behaviour of measurements at low signal-to-noise values. a) Elevation angle (degrees); b) L1 phase signal-to-noise (GPS receiver units); c) L1 P-code - L2 P-code rateof-change $(\mathrm{m} / \mathrm{s})$; d) L1 phase - L2 phase rate-of-change $(\mathrm{m} / \mathrm{s}) ; \mathbf{e})$ widelane phase - narrowlane pseudorange rate-of-change (m/s). GPS PRN08, day of year 148, 2001 

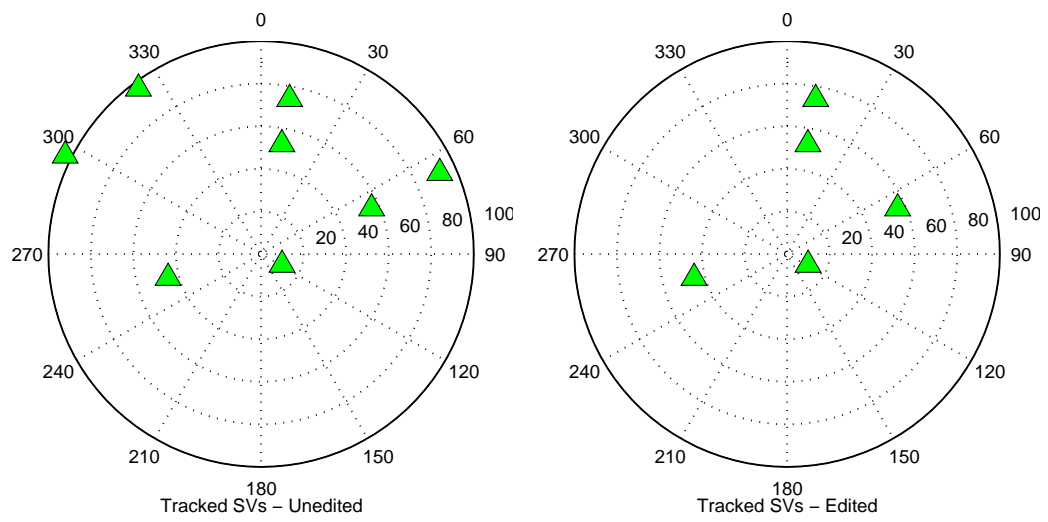

Fig. 4. Example of GPS satellite sky distribution before and after data editing. Day of year 148, 2001, 30.2389 hours. Azimuth of $180^{\circ} \approx$ velocity direction. Before editing: \# SVs $=8$ and $\mathrm{PDOP}=1.7$. After editing: $\# \mathrm{SVs}=5$ and $\mathrm{PDOP}=4.0$

\section{Results}

CHAMP data provided by the CHAMP Information System and Data Center (ISDC 2001) was processed for the period of the IGS LEO Orbit Comparison Campaign (ESOC 2001): day of year 140 to 150, 2001. The resulting UNB solutions were compared against Jet Propulsion Laboratory (JPL) solutions using a comparison interpolator developed by ESOC (ESOC 2001). Each 24 hour CHAMP data arc required less than 30 minutes for processing with UNB unoptimised computer code on an $85 \mathrm{MHz}$ Sun server.

Figure 5 shows the position component differences for day 143 - a typical day. The radial, along-track, cross-track, and norm r.m.s. (in $\mathrm{cm}$ ) are 36, 25, 24, and 50, respectively. The forward filter post-fit residual r.m.s. for the ionosphere-free pseudorange is $105 \mathrm{~cm}$ and for the time-difference ionosphere-free phase is $9 \mathrm{~cm}$. The spikes and much of the noise in the positions are due to data gaps and remaining poor-quality measurements. This is also evident from the relatively large postfit residuals.

Figure 6 illustrates the daily differences between UNB and JPL 24 hour arcs. The position difference r.m.s. ranges from: $30 \mathrm{~cm}$ to $57 \mathrm{~cm}$ in the radial direction; $21 \mathrm{~cm}$ to $39 \mathrm{~cm}$ along-track; $22 \mathrm{~cm}$ to $42 \mathrm{~cm}$ cross-track; and $43 \mathrm{~cm}$ to $76 \mathrm{~cm}$ for the norm. Notice the larger error difference in the radial component. The CHAMP radial component represents the nominal "up" component in the topocentric sense, and of course suffers from the GPS geometry predicament of having no transmitters below the receiver. The poor overall repeatability is again caused by the lack of continuous, quality GPS measurements. An indication of near-optimum solution comparison can be observed during periods of good continuous data, e.g., be- 
tween hours 2 and 3 of day of year 150. For this short arc, the r.m.s. differences between UNB and JPL are as small as: $13 \mathrm{~cm}, 10 \mathrm{~cm}, 7 \mathrm{~cm}$; in the radial, alongtrack, and cross-track directions, respectively, and $18 \mathrm{~cm}$ in the norm.

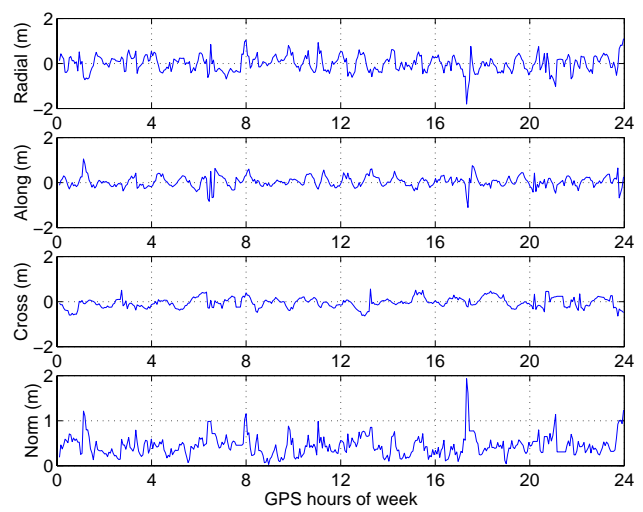

Fig. 5. Position component differences between UNB and JPL for day of year 143, 2001 r.m.s. $(\mathrm{cm})$ : radial $=36$; along-track $=25$; cross-track $=24$; norm $=50$. Filter post-fit residuals: ionosphere-free pseudorange $=105 \mathrm{~cm}$; time-difference ionosphere-free phase $=9 \mathrm{~cm}$

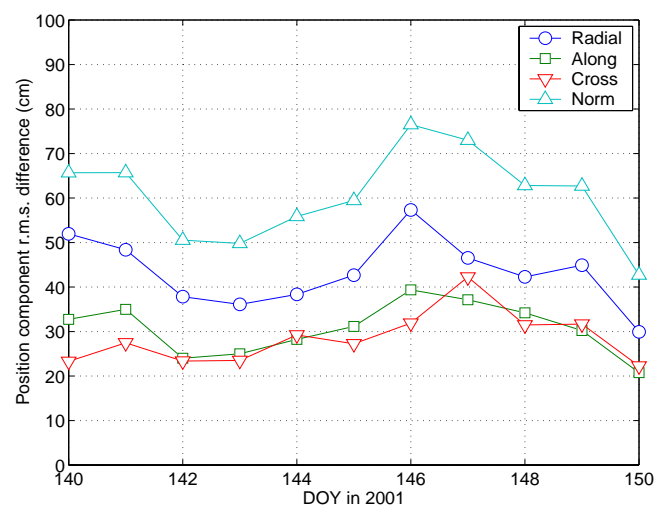

Fig. 6. Daily position component r.m.s. differences between UNB and JPL. Mean r.m.s. $(\mathrm{cm})$ : radial $=43$; along-track $=30$; cross-track $=28 ;$ norm $=60$

At the end of 2001, the CHAMP tracking algorithm was altered to prevent tracking of GPS satellites below the CHAMP antenna horizon. Such a situation would maximise the potential of the geometric solution strategy. From early analysis, this has resulted in a significant increase in the number of quality measurements, and tremendous reductions in position component r.m.s. differences 
with respect to dynamically determined orbits, with differences approaching the decimetre-level.

\section{Conclusions and Further Research}

An a posteriori LEO orbit determination strategy based solely on GPS measurements has been devised, which is simple and efficient. The processing strategy requires significant editing of the CHAMP GPS receiver measurements due to the receiver tracking algorithm. Intermediate results indicate 24 hour arc r.m.s. differences with respect to dynamical orbits of approximately $40 \mathrm{~cm}$ in the radial component and $30 \mathrm{~cm}$ in each of the along-track and cross-track components. Short arc r.m.s. differences as small as $10 \mathrm{~cm}$ in each component are possible. CHAMP single-receiver, GPS-only POD is attainable, but geometrically strong, continuous GPS measurements are required. Since only tens of minutes of processing time are required to process one day's worth of data, a real-time capability is possible.

A number of additional functional and stochastic modelling improvements will be made to improve the presented orbit solutions, and initial processing of recent CHAMP receiver data indicates quality datasets capable of providing neardecimetre-level precision with the UNB processing strategy.

\section{Acknowledgements}

This research was conducted with financial support from the Natural Sciences and Engineering Research Council of Canada and the GEOIDE Network of Centres of Excellence (Project ENV\#14). The authors would like to thank the IGS for having produced and made available the precise GPS satellite ephemerides and clock offset files.

\section{References}

Bisnath SB, Langley RB (1999) Precise A Posteriori Geometric Tracking of Low Earth Orbiters with GPS. Canadian Aeronautics and Space J 45: 245-252

Bisnath SB, Langley RB (2001) Precise Orbit Determination of Low Earth Orbiters with GPS Point Positioning. In: Proceedings of the Institute of Navigation 2001 National Technical Meeting, The Institute of Navigation, Alexandria, pp 725-733

ESOC (2001) IGS LEO Orbit Comparison Campaign 2001. <http://nng.esoc.esa.de/gps /campaign.html>. Accessed October 2001

GFZ (2001) CHAMP Home Page. <http://op.gfz-potsdam.de/champ/index_CHAMP.html>. Accessed January 2001

ISDC (2001) CHAMP Information System and Data Center. <http://isdc.gfz-potsdam.de /champ/>. Accessed October 2001 